\title{
Childhood hydrocephalus secondary to posterior fossa tumor is both an intra- and extraaxial process
}

\author{
Grant A. Bateman, MBBS, MD, FRANZCR,, ${ }^{1,2}$ and \\ Mark Fiorentino, MD, MMed(Radiol), FRCR, FRANZCR ${ }^{1}$ \\ ${ }^{1}$ Department of Medical Imaging, John Hunter Hospital; and ${ }^{2}$ Newcastle University Faculty of Health, Callaghan Campus, \\ Newcastle, New South Wales, Australia
}

\begin{abstract}
OBJECTIVE Ventricular dilation secondary to tumor obstruction of the posterior fossa CSF outflow in childhood is an intraaxial process. However, third ventriculostomy or complete tumor removal often fails to reduce the pressure in some children, and in others there is a delayed reduction in intracranial pressure; this is termed the adaptation period. The cause of this adaptation period has not been studied. Venous sinus compression is highly correlated with other forms of childhood hydrocephalus, and this study seeks to follow the changes that occur in sinus cross-sectional area after surgery.
\end{abstract}

METHODS Twelve children with posterior fossa tumors underwent MRI examination that included standard T2-weighted and 3D contrast-enhanced images obtained preoperatively, in the immediate postoperative period, and after several months. The volumes of the lateral and third ventricles and the minimum cross-sectional area of the transverse and sigmoid sinuses were measured. Patients were categorized by 1) shunt status (those who required a shunt vs those who did not) and 2) by age (those younger than 3 years vs those older than 3 years at diagnosis).

RESULTS There was a significant reduction in ventricular volume in both the immediate and secondary follow-up periods for all patients. There was preoperative venous sinus compression in all groups, which did not change significantly in the immediate postoperative period but did improve in the secondary follow-up period. The younger children had larger ventricles and smaller sinuses before surgery compared with the older children.

CONCLUSIONS In children with obstructed hydrocephalus caused by tumor, there is secondary compression of venous outflow, indicating both an intra- and extraaxial process. The expansion of the sinuses following decompression of the posterior fossa is delayed and may correlate with the adaptation period. Younger children have greater sinus compression than older ones.

http://thejns.org/doi/abs/10.3171/2016.1.PEDS15676

KEY WORDS obstructed hydrocephalus; adaptation period; children; tumor; venous sinus

$\mathrm{A}$ T presentation, children with posterior fossa tumors will be found to have hydrocephalus in $71 \%-90 \%$ of cases..$^{19,24}$ In the majority of these patients the hydrocephalus is evidently due to obstruction of the CSF outflow pathways through the aqueduct or fourth ventricle. This obstructive form of hydrocephalus is an intraaxial process. Highlighting the intraaxial nature of the process, tumor removal within 24 to 48 hours of admission in patients with symptomatic hydrocephalus brings relief from hydrocephalus in most cases. ${ }^{11}$ In one study, early total or subtotal tumor resection resolved the hydrocephalus in $91 \%$ of patients. ${ }^{16}$ Despite the overall success of surgery in unblocking the CSF pathways, the resolution of the CSF absorption abnormality is not always immediate. A review of the literature has suggested that the ventricles often remain enlarged in the initial period following surgery even in those who will improve. ${ }^{6}$ Due to the delay in improvement in most patients and the complete failure of hydrocephalus amelioration in some patients, endoscopic third ventriculostomy (ETV) has been advocated as a means of bypassing the blockage before tumor surgery. To further characterize the delayed improvement in hydrocephalus following surgery, Cinalli et al. monitored CSF pressure following ETV. A primary ETV was performed

ABBREVIATIONS CBF = cerebral blood flow; ETV = endoscopic third ventriculostomy; EVD = external ventricular drain; ICP = intracranial pressure.

SUBMITTED November 16, 2015. ACCEPTED January 13, 2016.

INCLUDE WHEN CITING Published online April 1, 2016; DOI: 10.3171/2016.1.PEDS15676. 
in 44 children, 31 of whom had obstruction secondary to posterior fossa tumor. In 52\% there was an initial high intracranial pressure (ICP) that progressively decreased; however, this process often took several days. In $29 \%$ of the children, there was an initial fall but subsequent rise in pressure, signaling failure of the procedure. ${ }^{6}$ This delay in improvement of the CSF pressure following the unblocking of the system has been called the adaptation period. It is thought that obstruction of the ventricular system may somehow cause a secondary reversible obstruction somewhere within the subarachnoid space ${ }^{15}-$ i.e., there is an extraaxial component to the intraaxial obstructed hydrocephalus. The exact cause of the extraaxial obstruction has not been elucidated. Previous work by one of the authors of the current study has shown evidence of collateral blood flow bypassing the venous outflow of the brain in a group of children whose mean age was 8 years and who had both obstructed and communicating hydrocephalus. This study suggested that a significant venous outflow stenosis was operating somewhere in these children. ${ }^{3}$ The exact site of this obstruction was not defined. The purpose of the current study is to measure the change in ventricular volume and in venous outflow sinus cross-sectional area in both the initial and delayed follow-up periods in children who have undergone surgery for posterior fossa tumor to further characterize the adaptation period.

\section{Methods \\ Patients}

The radiology information system at a tertiary referral hospital was retrospectively interrogated to locate all children younger than 10 years of age who presented with obstructed hydrocephalus secondary to a posterior fossa tumor from January 2010 to July 2015. Fourteen patients were found. In 2 patients, biopsy was performed, but neither shunt insertion nor excision of the tumor was attempted, and these patients were not investigated further. The remaining 12 patients, 7 females and 5 males, were included in the study. On T2-weighted imaging, all patients showed no evidence of flow void through the aqueduct secondary to obstruction of CSF outflow by tumor. The clinical findings are summarized in Table 1 . The average age of the patients at diagnosis was $4.1 \pm 3$ years. Six patients were less than 3 years old at diagnosis (average 1.7 \pm 0.7 years) and 6 were older (average $6.6 \pm 2.2$ years). Six patients had an external ventricular drain (EVD) inserted at the time of surgery. The remaining 6 had the tumor excised with restoration of CSF flow through the aqueduct into the basal cisterns and no EVD was inserted. None of the patients without an EVD subsequently required a shunt. In the 6 patients with an EVD, the drain was initially left on open drainage and set at $5-10 \mathrm{~cm} \mathrm{H}_{2} \mathrm{O}$. The drain was then progressively increased in height over several days such that it could be eventually clipped and removed, if possible. This removal occurred on average at postoperative Day 7. In these 6 patients, failure of EVD removal was heralded in all cases by either a prolonged increase in CSF pressure, a large volume of CSF drainage, or a CSF leak at the surgery site following removal. All 6 patients had a shunt subsequently inserted, on average at postoperative Day 17. Two patients had both a shunt and CSF outflow patency restored, but these were placed in the shunt group. The average interval before initial postoperative imaging was $1.8 \pm 1.6$ days after surgery and the secondary follow-up averaged $3.9 \pm 1.0$ months after surgery. There was no significant difference in age, sex, or follow-up period between the shunt and no shunt groups or between the patients younger and older than 3 years of age at diagnosis. There was no significant difference in shunt status between the younger and older patient groups. The study was approved by the hospital ethics committee, and was therefore performed in accordance with the ethical standards laid down in the 1964 Declaration of Helsinki.

\section{MRI Examination and Analysis}

Magnetic resonance imaging was performed in all patients using a 3.0-T superconducting magnet (Avanto, Seimens). Scanning included standard T2-weighted, FLAIR, and diffusion-weighted axial sequences as well as T1weighted 3D sagittal images before and after administration of intravenous contrast. Scanning was performed in the immediate preoperative period and as soon as practicable postoperatively to gauge the success of surgery. The next follow-up always occurred within 6 months according to the standard oncology protocols at our institution. The ventricular volume of the lateral and third ventricles was measured by tracing the outline of these structures on the T2-weighted images and multiplying the sum of the areas obtained by the slice thickness and any interslice gap to obtain the volume of the ventricles in milliliters. Using the 3D T1-weighted postcontrast data set, oblique multiplanar reconstructions were obtained along the course of the transverse and sigmoid sinuses in each patient to discover the narrowest segments. Reconstructions were then obtained perpendicular to the long axis of the sinus at the narrowest point and the cross-sectional area was measured with the work station measuring tool. The minimum cross-sectional areas for the right and left sinuses were added to obtain the total sinus area. The measurements were repeated at the same position as the preoperative imaging in the postoperative studies. Mean and standard deviations were obtained for each group. Differences between groups were tested using a MannWhitney U-test. The differences between paired samples were tested between preoperative and postoperative measurements using a Wilcoxon signed rank test. A 95\% confidence interval was used for all tests.

Figure 1 outlines the process for Patient 10. Panel A shows the degree of ventricular dilation occurring secondary to the posterior fossa tumor, noted in panel B. Panel $\mathrm{C}$ shows an MR venogram obtained at the initial study, which shows maximal narrowing at the distal sigmoid sinuses (arrows). The initial oblique coronal reconstruction along the plane of the sigmoid sinuses (panel D) shows the thinned contrast-containing sinuses compressed between the cerebellum and the inner table of the skull (arrows). Note the enhancing tumor and ventricular dilation. As the narrowest portion of the sinuses is distal, reconstructions were obtained at the levels of the vertical lines on each side. Panel E shows the right-side and panel $F$ the left-side reconstructions. The combined areas of the sinuses are 
TABLE 1. Clinical findings in 12 children

\begin{tabular}{ccllcccc}
\hline $\begin{array}{c}\text { Case } \\
\text { No. }\end{array}$ & $\begin{array}{c}\text { Age (yrs), } \\
\text { Sex }\end{array}$ & \multicolumn{1}{c}{ Tumor Type } & $\begin{array}{c}\text { Location of } \\
\text { Obstruction }\end{array}$ & $\begin{array}{c}\text { Shunt } \\
\text { Inserted }\end{array}$ & $\begin{array}{c}\text { Aqueduct Flow } \\
\text { Restored }\end{array}$ & $\begin{array}{c}\text { Initial FU } \\
\text { (days) }\end{array}$ & $\begin{array}{c}\text { Second FU } \\
\text { (mos) }\end{array}$ \\
\hline 1 & 0.9, M & Medulloblastoma & 4th ventricle & No & Yes & 1 & 4.0 \\
\hline 2 & $1.9, \mathrm{~F}$ & Ependymoma & 4th ventricle & No & Yes & 1 & 5.2 \\
\hline 3 & $1.3, \mathrm{~F}$ & Medulloblastoma & 4th ventricle & Yes & Yes & 2 & 3.9 \\
\hline 4 & $7.8, \mathrm{~F}$ & Pilocytic astrocytoma & 4th ventricle & No & Yes & 2 & 3.2 \\
\hline 5 & $2.9, \mathrm{~F}$ & Ganglioneuroblastoma & Aqueduct & Yes & No & 1 & 3.9 \\
\hline 6 & $5.9, \mathrm{M}$ & Medulloblastoma & 4th ventricle & Yes & No & 1 & 1.4 \\
\hline 7 & $9.5, \mathrm{~F}$ & Glioma & 4th ventricle & No & Yes & 6 & 4.8 \\
\hline 8 & $1.5, \mathrm{M}$ & Pilocytic astrocytoma & Aqueduct & No & Yes & 1 & 5.4 \\
\hline 9 & $3.3, \mathrm{~F}$ & Astrocytoma & Aqueduct & No & Yes & 1 & 3.3 \\
\hline 10 & $1.8, \mathrm{M}$ & Pilocytic astrocytoma & 4th ventricle & Yes & No & 1 & 4.0 \\
\hline 11 & $5.5, \mathrm{M}$ & Medulloblastoma & 4th ventricle & Yes & No & 1 & 4.0 \\
\hline 12 & $7.3, \mathrm{~F}$ & Medulloblastoma & 4th ventricle & Yes & Yes & 4 & 3.9 \\
\hline Mean & 4.1 & & & & & 1.8 & 3.9 \\
\hline SD & 3.0 & & & & & 1.6 & 1.0 \\
\hline
\end{tabular}

$\mathrm{FU}=$ follow-up.

just over $22 \mathrm{~mm}^{2}$. Immediately following surgery (panel $\mathrm{G})$, the center of the tumor has been debulked and the subarachnoid space around the cerebellum restored (arrows). An EVD was inserted and the ventricles became smaller. The coronal reconstruction along the sigmoid sinuses shows some mild improvement proximally, but no obvious change in size distally. Panels $\mathrm{H}$ and I are taken at the same points as $\mathrm{E}$ and $\mathrm{F}$ and confirm no significant change in size. At 4 months postsurgery, the ventricles are reduced further in size (panel $\mathrm{J}$ ) and the sinuses have enlarged fully into the available subarachnoid space (panels $\mathrm{K}$ and $\mathrm{L}$ ) with a combined sinus area of $105 \mathrm{~mm}^{2}$ (a 500\% increase).

\section{Results}

Taken as a group, in the 12 patients, the preoperative average ventricular volume was $111 \pm 68 \mathrm{ml}$; at the initial follow-up it decreased to $74 \pm 47 \mathrm{ml}$ (a $33 \%$ reduction in size; $p=0.003$ ), with a further reduction in size to $42 \pm$ $36 \mathrm{ml}$ at the second follow-up (a 62\% reduction compared with the initial volume; $\mathrm{p}=0.003$ ). The average preoperative sinus area for the group was $27 \pm 18 \mathrm{~mm}^{2}$. At the initial follow-up the sinus area was not significantly different at $34 \pm 28 \mathrm{~mm}^{2}$. At the second follow-up the area of the sinuses had increased to $80 \pm 28 \mathrm{~mm}^{2}$ (a $300 \%$ increase in size; $p=0.002$ ).

The ventricular and sinus findings for the 2 groups (those who received shunts and those who did not) are summarized in Table 2. There was a trend for the ventricles to be larger in the shunt group than in the nonshunt group preoperatively (ventricle size is a risk factor for shunt), but this was not statistically significant. At the second follow-up there was a trend for the sinuses to be larger in the shunt group, but this also failed to be significant. All other measurements compared between the shunt and nonshunt groups were not significantly different.

The imaging findings for the age groups (less than vs greater than age 3 years) are summarized in Table 3 . When stratified for age, those under 3 years at diagnosis had an initial average ventricular volume of $163 \pm 39 \mathrm{ml}$, which was larger than the ventricular volume found in the older group $(61 \pm 43 \mathrm{ml} ; \mathrm{p}=0.02)$. The difference in ventricular volumes became reduced over time, with no significant difference with age being found at the first and subsequent follow-ups. This is shown graphically in Fig. 2 upper. The average preoperative sinus area in those under 3 years was $14 \pm 5 \mathrm{ml}$, and in those older than 3 years it was $41 \pm 16$ $\mathrm{ml}$, a significant difference $(\mathrm{p}=0.008)$. There was no significant difference at the initial follow-up or at the second follow-up in the sinus areas between these groups as they changed over time. These findings are shown graphically in Fig. 2 lower.

\section{Discussion}

There is a significant correlation between the development of hydrocephalus and posterior fossa tumor in children. In one study, despite similar degrees of CSF outflow obstruction, 10 patients presented without hydrocephalus, 32 had mild hydrocephalus, 14 had moderate, and 8 had marked hydrocephalus. ${ }^{7}$ The normal ventricular volume in childhood is $21 \mathrm{ml}^{25}$ It can be seen from Table 2 and Fig. 2 that the current study, like others described in the literature, contains patients with ventricles ranging from mildly to severely dilated. Even after tumor surgery, up to $30 \%$ of children will have residual hydrocephalus requiring a shunt postoperatively. ${ }^{18}$ The risk factors associated with the need for a postoperative shunt to control hydrocephalus are age ( $<3$ years), midline tumor location, subtotal resection, pseudomeningocele formation, and CSF infection. ${ }^{7}$ Other risk factors include larger ventricles at presentation ${ }^{21}$ and tumor type. Medulloblastoma and ependymoma require shunt placement more frequently than do astrocytomas. ${ }^{9}$ In the current study, trial removal of an EVD failed in $50 \%$ of the patients and shunt insertion was 

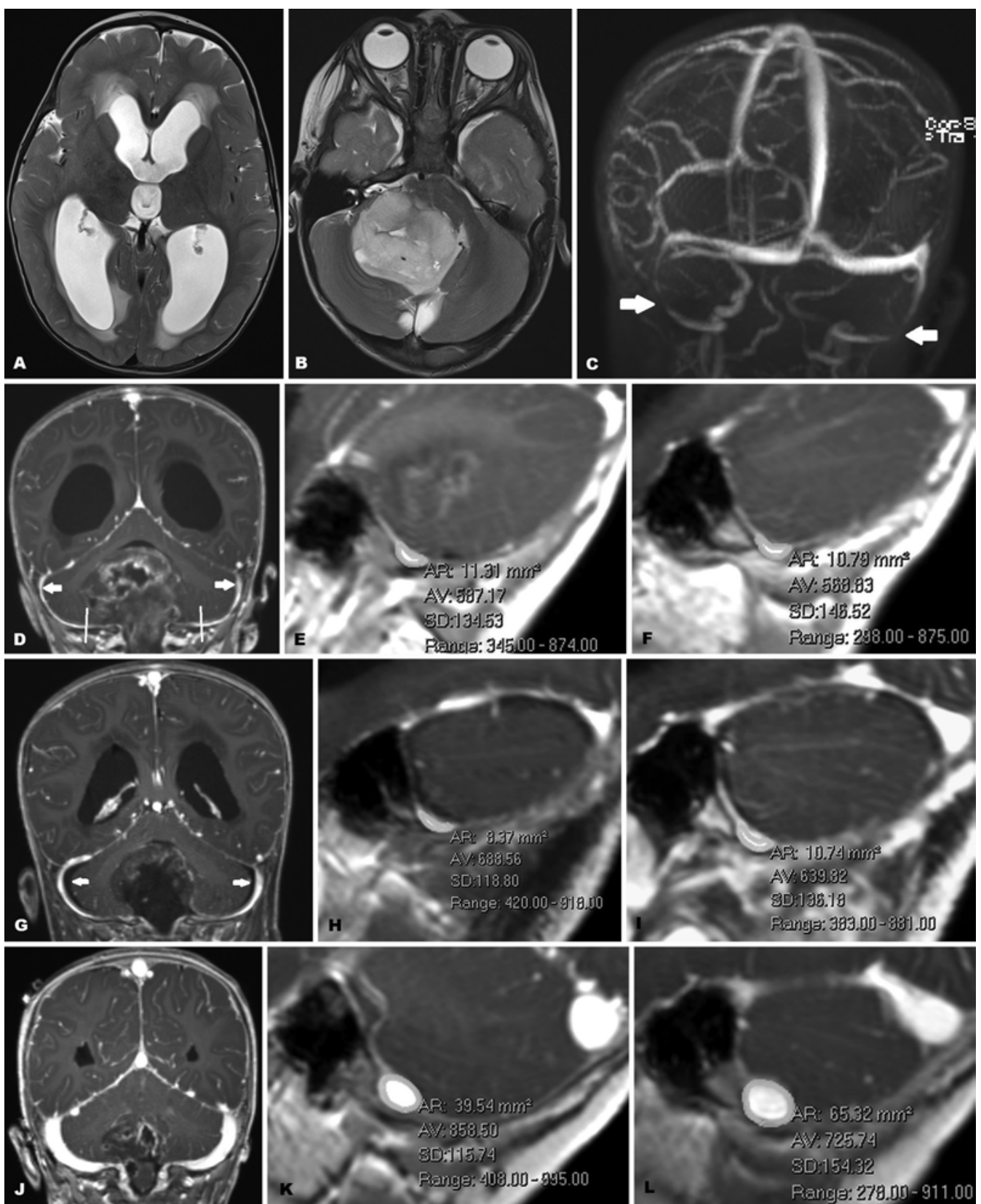

FIG. 1. Patient 10. A: T2-weighted axial preoperative image showing triventricular enlargement and subependymal edema. B: T2-weighted axial image showing the obstructing tumor in the cerebellum. C: MR venogram obtained preoperatively viewed from behind with arrows showing stenosis of both sigmoid sinuses. D: Preoperative oblique coronal reconstruction along the sigmoid sinuses with arrows showing the compressed contrast-enhanced sinuses between the cerebellum and the inner table of the skull on both sides. The short vertical lines show the position and orientation of the perpendicular reconstructions of the sinuses. Note the central tumor and the ventricular dilation. E: Reconstruction of the right transverse sinus showing the area of the sinus to be $11.31 \mathrm{~mm}^{2}$. F: Reconstruction of the left transverse sinus showing the area of the sinus to be $10.79 \mathrm{~mm}^{2}$. G: Initial follow-up oblique coronal reconstruction along the sigmoid sinuses showing debulking of the tumor and reconstitution of the posterior fossa subarachnoid space (arrows). The ventricles are smaller. The proximal sigmoid sinuses have minimally increased in size, but the distal sinuses remain unchanged. $\mathrm{H}$ : Initial postoperative reconstruction of the right transverse sinus taken at the same point as panel $E$ shows the area of the sinus to be not significantly different. I: Initial postoperative reconstruction of the left transverse sinus taken at the same point as panel $\mathrm{F}$ shows the area of the sinus to be not significantly different. J: Second follow-up oblique coronal reconstruction along the sigmoid sinuses shows some residual tumor and a further reduction in the ventricles. The sigmoid sinuses have fully expanded into the available subarachnoid space. K: Reconstruction of the right transverse sinus shows the area of the sinus to be $39.54 \mathrm{~mm}^{2}$. L: Reconstruction of the left transverse sinus shows the area of the sinus to be $65.32 \mathrm{~mm}^{2}$. 
TABLE 2. Imaging findings in children with shunts and those with patent aqueducts*

\begin{tabular}{|c|c|c|c|c|c|c|c|}
\hline \multirow[b]{2}{*}{ Case No. \& Group } & \multicolumn{3}{|c|}{ Ventricular Vol (ml) } & \multirow{2}{*}{$\begin{array}{c}\text { Sinus Stenosis } \\
\text { Site }\end{array}$} & \multicolumn{3}{|c|}{ Sinus Area $\left(\mathrm{mm}^{2}\right)$} \\
\hline & Preop & Initial FU & 2nd FU & & Preop & Initial FU & 2nd FU \\
\hline \multicolumn{8}{|l|}{ Shunt inserted } \\
\hline 3 & 206 & 73 & 27 & Sigmoid & 17 & 52 & 78 \\
\hline 5 & 137 & 96 & 3 & Transverse & 6 & 19 & 116 \\
\hline 6 & 77 & 60 & 21 & Transverse & 32 & 24 & 73 \\
\hline 10 & 206 & 130 & 37 & Sigmoid & 22 & 19 & 105 \\
\hline 11 & 138 & 119 & 77 & Sigmoid & 41 & 35 & 83 \\
\hline 12 & 55 & 10 & 48 & Sigmoid & 56 & 67 & 83 \\
\hline Mean & $137 \pm 63$ & $81 \pm 43$ & $36 \pm 25$ & & $29 \pm 17$ & $36 \pm 19$ & $90 \pm 17$ \\
\hline \multicolumn{8}{|l|}{ No shunt inserted } \\
\hline 1 & 176 & 150 & 100 & Sigmoid & 14 & 32 & 45 \\
\hline 2 & 139 & 102 & 32 & Transverse & 14 & 41 & 136 \\
\hline 4 & 30 & 24 & 15 & Transverse & 19 & 17 & 72 \\
\hline 7 & 17 & 21 & 20 & Transverse & 37 & 35 & 49 \\
\hline 8 & 116 & 88 & 80 & Sigmoid & 11 & 21 & 51 \\
\hline 9 & 33 & 17 & 14 & Sigmoid & 61 & 65 & 68 \\
\hline Mean & $85 \pm 67$ & $67 \pm 55$ & $43 \pm 37$ & & $26 \pm 20$ & $35 \pm 17$ & $70 \pm 34$ \\
\hline$p$ value & 0.23 & 0.81 & 0.94 & & 0.69 & 1.00 & 0.06 \\
\hline
\end{tabular}

* Mean values are presented $\pm S D ; p$ values are for comparison of group means.

required; 4 of these 6 children had medulloblastomas. In 2 patients who received a shunt, the flow of CSF through the aqueduct into the basal cisterns was restored, but in 4 it was not. Those requiring a shunt tended to have larger ventricles, correlating well with the risk factors suggesting the possible need for a shunt, as previously discussed.
In the present study, 6 patients received an EVD, which was set to drain at a height of 5-10 $\mathrm{cm} \mathrm{H}_{2} \mathrm{O}$, and this drainage occurred during the 2 days before the initial follow-up (the average drain removal was at postoperative Day 7). A trial of EVD removal or clipping failed in all 6, and a shunt was inserted on average on postoperative Day 17 (well be-

TABLE 3. Imaging findings in children younger and older than 3 years*

\begin{tabular}{|c|c|c|c|c|c|c|c|}
\hline \multirow[b]{2}{*}{ Case No. \& Group } & \multicolumn{3}{|c|}{ Ventricular Vol (ml) } & \multirow{2}{*}{$\begin{array}{c}\text { Sinus Stenosis } \\
\text { Site }\end{array}$} & \multicolumn{3}{|c|}{ Sinus Area $\left(\mathrm{mm}^{2}\right)$} \\
\hline & Preop & Initial FU & 2nd FU & & Preop & Initial FU & 2nd FU \\
\hline \multicolumn{8}{|c|}{ Findings in children younger than 3 years } \\
\hline 1 & 176 & 150 & 100 & Sigmoid & 14 & 32 & 45 \\
\hline 2 & 139 & 102 & 32 & Transverse & 14 & 41 & 136 \\
\hline 3 & 206 & 73 & 27 & Sigmoid & 17 & 52 & 78 \\
\hline 5 & 137 & 96 & 3 & Transverse & 6 & 19 & 116 \\
\hline 8 & 116 & 88 & 80 & Sigmoid & 11 & 21 & 51 \\
\hline 10 & 115 & 10 & 114 & Sigmoid & 22 & 67 & 105 \\
\hline Mean & $163 \pm 39$ & $81 \pm 43$ & $52 \pm 44$ & & $14 \pm 5$ & $36 \pm 19$ & $88 \pm 36$ \\
\hline \multicolumn{8}{|c|}{ Findings in children older than 3 yrs } \\
\hline 4 & 30 & 24 & 15 & Transverse & 19 & 17 & 72 \\
\hline 6 & 77 & 60 & 21 & Transverse & 32 & 24 & 73 \\
\hline 7 & 17 & 21 & 20 & Transverse & 37 & 35 & 49 \\
\hline 9 & 33 & 17 & 14 & Sigmoid & 61 & 65 & 68 \\
\hline 11 & 138 & 119 & 77 & Sigmoid & 41 & 35 & 83 \\
\hline 12 & 55 & 10 & 48 & Sigmoid & 56 & 67 & 83 \\
\hline Mean & $61 \pm 43$ & $67 \pm 55$ & $34 \pm 24$ & & $41 \pm 16$ & $35 \pm 17$ & $71 \pm 12$ \\
\hline$p$ value & $0.02 \dagger$ & 0.20 & 0.23 & & $0.008 \dagger$ & 1.00 & 0.47 \\
\hline
\end{tabular}

${ }^{*}$ Mean values are presented $\pm S D$.

$\dagger p$ value $<0.05$. 
Change in Ventricle Volume vs Age

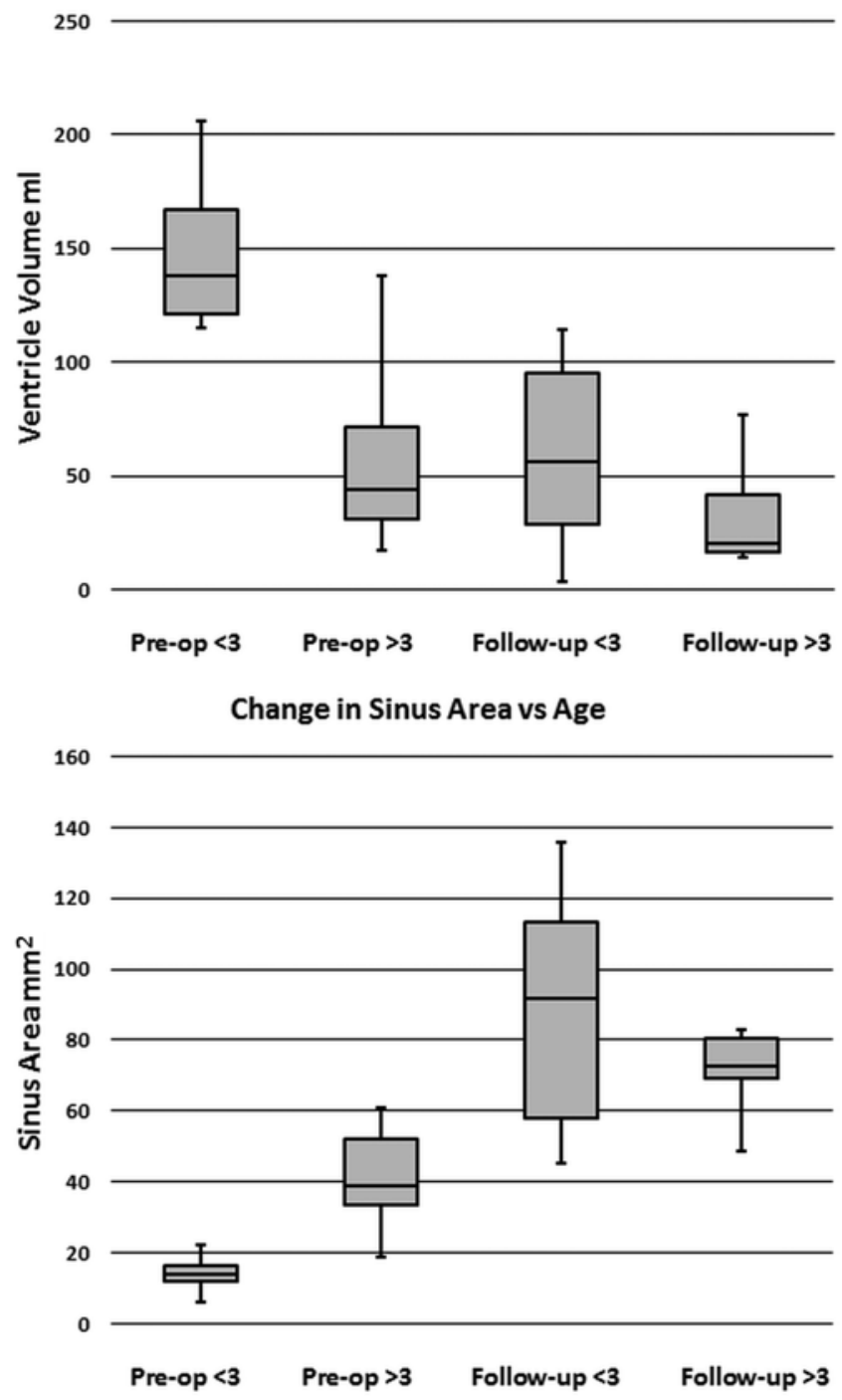

FIG. 2. Upper: Box and whiskers plot comparing the change in ventricle size between the preoperative and second follow-up studies in children younger or older than 3 years. Lower: Box and whiskers plot comparing the change in sinus area between the preoperative and second follow-up studies in children younger or older than 3 years.

fore the second follow-up at 3.9 months). The remaining 6 patients had no drainage and presumably had higher ICPs at both the initial and delayed follow-up studies. Despite this there was no significant difference between groups, either in the size of the ventricles or the sinuses at the initial or subsequent follow-up. Thus, CSF drainage did not appear to be a significant confounder in this study. The age at diagnosis had a much larger effect. Table 3 and Fig. 2 show the ventricles of the younger children to be much larger than those of the older patients preoperatively. This is presumably due to the more compliant sutures in younger children, which allow greater skull expansion and therefore more capacious ventricles. Despite this, the ventricle sizes in the 2 age groups were similar at both follow-up intervals. Similarly, the area of the sinuses was $66 \%(\mathrm{p}=$
0.008) smaller in the younger patients in comparison with the older patients in the preoperative study, but this difference was also lost at follow-up. The findings may indicate that the sinus walls in younger children are more compliant than those in the older cohort.

\section{The Adaptation Period}

As previously discussed, debulking a posterior fossa tumor and restoring the CSF flow does not immediately improve the degree of ventricular dilation or reduce the CSF pressure in the majority of patients. ${ }^{6}$ This period of delay has been termed the adaptation period. The cause of the adaptation period has been hypothesized to be progressive reopening of the subarachnoid spaces at the convexity, a slow increase in permeability of the pacchionian granulations, or possibly effusion of the CSF into the spinal subdural space. ${ }^{5}$ Hirsch et al. noted the ICP could remain high and only progressively return to normal after ventriculocisternostomy and attributed this finding to progressive opening of the subarachnoid spaces. ${ }^{12}$ The suggestion that the progressive opening of the subarachnoid spaces accounts for the adaptation period does not correlate well with our findings. As previously discussed, the adaptation period often lasts for several days, but may last as long as 2 weeks. Highlighting the delay, in 15 patients treated for failed shunt, $80 \%$ of whom had obstruction secondary to tumor, half had low CSF volumes passing from an EVD set at $30 \mathrm{~cm}$ and the other half had high flow for the first few days, which decreased gradually over the course of 1-2 weeks. ${ }^{17}$ The increase in the volume of the subarachnoid space after surgery occurs much quicker than this. In the present study, debulking of the tumor always improved the basal cistern volume at Day 2 (see Fig. 1 as an example). In addition, there was a $33 \%(\mathrm{p}=0.003)$ reduction in the ventricular volume supratentorially within 2 days of surgery. This finding is very similar to the findings of Di Rocco et al. Following ETV, ventricular volumes in children were found to reduce on average by $24 \%$ of the original volume by Day $3 .{ }^{8}$ Di Rocco et al. also noted that the volume of the subarachnoid space increased to $192 \%$ of the original by Day $3^{8}$ and, given the low viscosity of CSF, such a change in subarachnoid space volume would not continue to offer a high enough resistance to CSF flow to account for the adaptation period extending from several days to 2 weeks. Another group performed a trial of lumbar puncture and removal of 5-10 $\mathrm{ml}$ of CSF in children with a prolonged elevation in CSF pressure. The positive effect of the CSF removal was longer-lasting than can be explained by the small amount of CSF removed. In most cases, the ICP values remained normal or normalized after several lumbar punctures. ${ }^{6}$ Thus, the length of the adaptation period does not correlate with the opening of the subarachnoid space. In addition, it is hard to conceive how a lumbar puncture could improve the permeability of the pacchionian granulations or the absorption into the subdural space because reducing the CSF pressure should have the opposite effect. However, lumbar puncture has been found to improve venous sinus cross-sectional area in patients with idiopathic intracranial hypertension, reducing venous and CSF pressure $^{4}$ and perhaps accounting for this effect, as discussed later. 


\section{Sinus Cross-Sectional Area}

The normal venous sinus cross-sectional area obtained by adding the areas of the left and right mid-transverse sinuses is $72 \mathrm{~mm},{ }^{2}$ and this does not change significantly from the 1st to the 5 th decades of life. ${ }^{2}$ Following restoration of the CSF outflow, review at 4 months showed that the sinus area, $70 \mathrm{~mm}^{2}$, found in the nonshunt patients was not significantly different from normal. Sinus area in the shunt group showed a trend to be somewhat larger than normal at $90 \mathrm{~mm}^{2}(\mathrm{p}=0.06)$, but this may relate to a lower than normal CSF pressure that allows the veins to overdilate when a shunt is used. Preoperatively, the sinuses were much smaller than normal, with the average for the entire group being $27 \mathrm{~mm}^{2}$, which would equate to an overall $66 \%$ cross-sectional stenosis of the venous sinus. The degree of stenosis is dependent on age; Fig. 2 lower shows a much smaller sinus size in those under 3 years compared with those over 3 years in the preoperative study. The average sinus in children under 3 years was $14 \mathrm{~mm}^{2}$, which would be the equivalent of an $84 \%$ stenosis by area compared with the final sinus size for the group. Such a highgrade stenosis has been correlated with cerebral oligemia in neonates with hydrocephalus ${ }^{1}$ and is known to elevate sinus pressure and therefore CSF pressure in adults with idiopathic intracranial hypertension. ${ }^{10}$ In 19 patients (mean age 6.5 years) presenting with hydrocephalus secondary to a posterior fossa tumor, Yeom et al. showed an overall reduction of $45 \%$ in cerebral blood flow (CBF) compared with controls. The CBF increased to normal by 27 days after surgery. ${ }^{26}$ These authors considered the oligemia to be due to vascular compression secondary to a raised ICP, but did not comment on the site of compression. Could the venous sinus stenosis, noted in the present study, account for the reduction in blood flow? According to Poiseuille's equation, the pressure drop across a tube is proportional to the flow through the tube and inversely proportional to the square of the cross-sectional area of the tube (the length of the sinuses and the blood viscosity remain constant in hydrocephalus). ${ }^{2}$ If the pressure drop across a sinus was 1 $\mathrm{mm} \mathrm{Hg}$ and the flow through it was $400 \mathrm{ml} /$ minute and its initial area was $72 \mathrm{~mm}^{2}$, then reducing the flow by $45 \%$ on its own (i.e., a residual flow of $220 \mathrm{ml} /$ minute) would reduce the pressure drop to $0.55 \mathrm{~mm} \mathrm{Hg}$. Reducing the cross-sectional area by $84 \%$ on its own (i.e. a residual of $11.5 \mathrm{~mm}^{2}$ ) would increase the pressure drop to $39 \mathrm{~mm} \mathrm{Hg}$. Given that the normal pressure drop from the sagittal sinus to the jugular bulb is $4.5 \mathrm{~mm} \mathrm{Hg}$ in children, ${ }^{2}$ even if the average reduction in the size of the sinuses were only $50 \%$ from the sagittal sinus to the jugular bulb (the current study measures the maximal stenosis, not the average stenosis), the pressure increase in the sinuses would be 10 $\mathrm{mm} \mathrm{Hg}$. This correlates with the findings of Cinalli et al., who found the average initial CSF pressure to be $10 \mathrm{~mm}$ $\mathrm{Hg}$ higher than normal in children with posterior fossa tumor $^{6}$ (CSF pressure correlates directly with sinus pressure in idiopathic intracranial hypertension with venous stenosis $\left.{ }^{10}\right)$. In a study undertaken in children with hydrocephalus by Shulman and Ransohoff, in the 8 patients with hydrocephalus secondary to benign aqueduct stenosis, the sagittal sinus pressures averaged $16.1 \mathrm{~mm} \mathrm{Hg}$ and the CSF pressure averaged $13.6 \mathrm{~mm} \mathrm{Hg.}{ }^{22}$ Note the loss of the pres- sure gradient across the arachnoid granulations required for CSF absorption in this study. This finding has been assumed to be unimportant (i.e., the sinus pressure was found to be entirely secondary to the ICP rise) because in a subsequent study, the sinus pressures were shown to rapidly drop to the jugular bulb pressure once CSF was removed. However, this change required that the ICP be dropped to zero. ${ }^{20}$ Perhaps some delay in the reduction in sinus pressure might have been noted had the ICP been left at physiological levels or reduced slowly in this study, as occurs when posterior fossa tumor surgery is performed, rather than rapidly dropped to zero.

Therefore, delayed reexpansion of the venous sinuses within the tight posterior fossa caused by the expanding tumor mass could be the cause of the prolonged increased CSF pressure in the adaptation period. From the current data it is noted that the venous sinuses failed to significantly change in size at 2 days even with an EVD moderately lowering the pressure around the sinuses. At 4 months, the sinuses had all expanded into the subarachnoid space, with no residual stenosis noted. Thus, the venous and CSF pressure would have moderated sometime after 2 days but before 4 months due to resolution of the sinus stenosis. This correlates with the expected time frame of the adaptation period. One may ask, why do the sinuses not rapidly expand once the ICP is reduced? If the walls of the sinuses were perfectly elastic, then the stress of compression would be stored as strain within the walls themselves, and once the stress was removed the walls would bounce back. However, most biological tissues are viscoelastic and the viscous properties would allow plastic deformation of the walls to reduce the strain. Thus, the walls would take some time to readjust once the stress is removed. In the same way, the brain will not immediately reexpand once a long-standing mass such as a chronic subdural hematoma is removed, but may take several days..$^{13}$

These findings may also shed some light on why there is an age-related success rate for third ventriculostomy. A much higher rate of ETV failure has been found in children younger than 1 year compared with older children. ${ }^{14}$ In a review of 19 publications, the mean success rate for third ventriculostomy for children under 2 years of age was $48 \%{ }^{14,23}$ Finally, after tumor removal, $68 \%$ of children younger than 3 years required a shunt; shunts were required in only $32 \%$ of children older than 3 years. ${ }^{7}$ The explanation of the age-related success of ETV would appear to be that the sinus walls of younger children are more compliant and less able to withstand compression, leading to higher venous and CSF pressures and possibly a longer time to resolution of the pressure changes with a longer adaptation period. As previously discussed, the sinuses of children are so compressible that they can even be rendered oligemic by a venous outflow stenosis. ${ }^{1,26}$ The successful trial of lumbar puncture by Cinalli et al. in those with a prolonged adaptation period raises the possibility that perhaps an EVD should be drained at subphysiological pressures in the first few days after surgery to encourage a more rapid reexpansion of the sinuses as opposed to the current practice of rapidly elevating the pressure, which tries to enlarge the subarachnoid spaces or open the arachnoid granulations. 


\section{Conclusions}

In children with obstructed hydrocephalus caused by tumor, there is secondary compression of the venous outflow, which appears to be due to direct compression of the sinuses between the cerebellum and the skull. Thus, the hydrocephalus is both an intra- and extraaxial process. The expansion of the sinuses following decompression of the posterior fossa is delayed and may correlate with the adaptation period. Younger children have a greater degree of sinus compression, perhaps correlating with the poor response to third ventriculostomy in younger age groups. These findings suggest that further investigation into the venous sinus outflow changes in obstructed hydrocephalus is warranted as it may be of benefit in predicting prognosis of the hydrocephalus following surgery.

\section{References}

1. Bateman GA: Hemodynamically significant venous collapse underlying neonatal hydrocephalus. J Neurosurg Pediatr 13:125-132, 2014

2. Bateman GA, Siddique SH: Cerebrospinal fluid absorption block at the vertex in chronic hydrocephalus: obstructed arachnoid granulations or elevated venous pressure? Fluids Barriers CNS 11:11, 2014

3. Bateman GA, Smith RL, Siddique SH: Idiopathic hydrocephalus in children and idiopathic intracranial hypertension in adults: two manifestations of the same pathophysiological process? J Neurosurg 107 (6 Suppl):439-444, 2007

4. Bateman GA, Stevens SA, Stimpson J: A mathematical model of idiopathic intracranial hypertension incorporating increased arterial inflow and variable venous outflow collapsibility. J Neurosurg 110:446-456, 2009

5. Cartmill M, Vloeberghs M: The fate of the cerebrospinal fluid after neuroendoscopic third ventriculostomy. Childs Nerv Syst 16:879-881, 2000

6. Cinalli G, Spennato P, Ruggiero C, Aliberti F, Zerah M, Trischitta V, et al: Intracranial pressure monitoring and lumbar puncture after endoscopic third ventriculostomy in children. Neurosurgery 58:126-136, 2006

7. Culley DJ, Berger MS, Shaw D, Geyer R: An analysis of factors determining the need for ventriculoperitoneal shunts after posterior fossa tumor surgery in children. Neurosurgery 34:402-408, 1994

8. Di Rocco F, Grevent D, Drake JM, Boddaert N, Puget S, Roujeau T, et al: Changes in intracranial CSF distribution after ETV. Childs Nerv Syst 28:997-1002, 2012

9. Due-Tønnessen BJ, Helseth E: Management of hydrocephalus in children with posterior fossa tumors: role of tumor surgery. Pediatr Neurosurg 43:92-96, 2007

10. Fargen KM, Velat GJ, Lewis SB, Hoh BL, Mocco J, Lawson MF: Concomitant intracranial pressure monitoring during venous sinus stenting for intracranial hypertension secondary to venous sinus stenosis. J Neurointerv Surg 5:e22, 2013

11. Fritsch MJ, Doerner L, Kienke S, Mehdorn HM: Hydrocephalus in children with posterior fossa tumors: role of endoscopic third ventriculostomy. J Neurosurg 103 (1 Suppl):40-42, 2005

12. Hirsch JF, Hirsch E, Sainte Rose C, Renier D, Pierre-Khan A: Stenosis of the aqueduct of Sylvius. Etiology and treatment. J Neurosurg Sci 30:29-39, 1986

13. Kudoh C, Sugiura K, Kunimoto K, Takizawa H: Postoperative morphological recovery of deformed brain in cases of chronic subdural hematoma, in Nakamura N, Hashimoto T, Yasue M (eds): Recent Advances in Neurotraumatology. Tokyo: Springer, 1993, pp 149-152
14. Lam S, Harris D, Rocque BG, Ham SA: Pediatric endoscopic third ventriculostomy: a population-based study. J Neurosurg Pediatr 14:455-464, 2014

15. Milhorat TH, Hammock MK, Di Chiro G: The subarachnoid space in congenital obstructive hydrocephalus. 1. Cisternographic findings. J Neurosurg 35:1-6, 1971

16. Morelli D, Pirotte B, Lubansu A, Detemmerman D, Aeby A, Fricx C, et al: Persistent hydrocephalus after early surgical management of posterior fossa tumors in children: is routine preoperative endoscopic third ventriculostomy justified? J Neurosurg 103 (3 Suppl):247-252, 2005

17. Nishiyama K, Mori H, Tanaka R: Changes in cerebrospinal fluid hydrodynamics following endoscopic third ventriculostomy for shunt-dependent noncommunicating hydrocephalus. J Neurosurg 98:1027-1031, 2003

18. Riva-Cambrin J, Detsky AS, Lamberti-Pasculli M, Sargent MA, Armstrong D, Moineddin R, et al: Predicting postresection hydrocephalus in pediatric patients with posterior fossa tumors. J Neurosurg Pediatr 3:378-385, 2009

19. Sainte-Rose C, Cinalli G, Roux FE, Maixner R, Chumas PD, Mansour M, et al: Management of hydrocephalus in pediatric patients with posterior fossa tumors: the role of endoscopic third ventriculostomy. J Neurosurg 95:791-797, 2001

20. Sainte-Rose C, LaCombe J, Pierre-Kahn A, Renier D, Hirsch JF: Intracranial venous sinus hypertension: cause or consequence of hydrocephalus in infants? J Neurosurg 60:727736,1984

21. Santos de Oliveira R, Barros Jucá CE, Valera ET, Machado HR: Hydrocephalus in posterior fossa tumors in children. Are there factors that determine a need for permanent cerebrospinal fluid diversion? Childs Nerv Syst 24:1397-1403, 2008

22. Shulman K, Ransohoff J: Sagittal sinus venous pressure in hydrocephalus. J Neurosurg 23:169-173, 1965

23. Sufianov AA, Sufianova GZ, Iakimov IA: Endoscopic third ventriculostomy in patients younger than 2 years: outcome analysis of 41 hydrocephalus cases. J Neurosurg Pediatr 5:392-401, 2010

24. Taylor WA, Todd NV, Leighton SE: CSF drainage in patients with posterior fossa tumours. Acta Neurochir (Wien) 117:16, 1992

25. Xenos C, Sgouros S, Natarajan K: Ventricular volume change in childhood. J Neurosurg 97:584-590, 2002

26. Yeom KW, Lober RM, Alexander A, Cheshier SH, Edwards MSB: Hydrocephalus decreases arterial spin-labeled cerebral perfusion. AJNR Am J Neuroradiol 35:1433-1439, 2014

\section{Disclosures}

The authors report no conflict of interest concerning the materials or methods used in this study or the findings specified in this paper.

\section{Author Contributions}

Conception and design: Bateman. Acquisition of data: both authors. Analysis and interpretation of data: Bateman. Drafting the article: Bateman. Critically revising the article: both authors. Reviewed submitted version of manuscript: both authors. Approved the final version of the manuscript on behalf of both authors: Bateman. Statistical analysis: Bateman. Administrative/ technical/material support: Bateman. Study supervision: Bateman.

\section{Correspondence}

Grant Bateman, Department of Medical Imaging, John Hunter Hospital, Locked Bag 1, Newcastle Region Mail Center, Newcastle, NSW 2310, Australia. email: grant.bateman@ hnehealth.nsw.gov.au. 